\title{
Publisher's Note: Positronium-atom scattering at low energies [Phys. Rev. A 90, 052717 (2014)]
}

\author{
I. I. Fabrikant and G. F. Gribakin
}

(Received 20 December 2017; published 17 January 2018)

DOI: 10.1103/PhysRevA.97.019903

This paper was published online on 20 November 2014 with errors in the positioning of the images of Figs. 3 and 8 . The figures have been corrected as of 19 December 2017. The figures are incorrect in the printed version of the journal; therefore, for the benefit of the print readership, the figures have been replicated below.

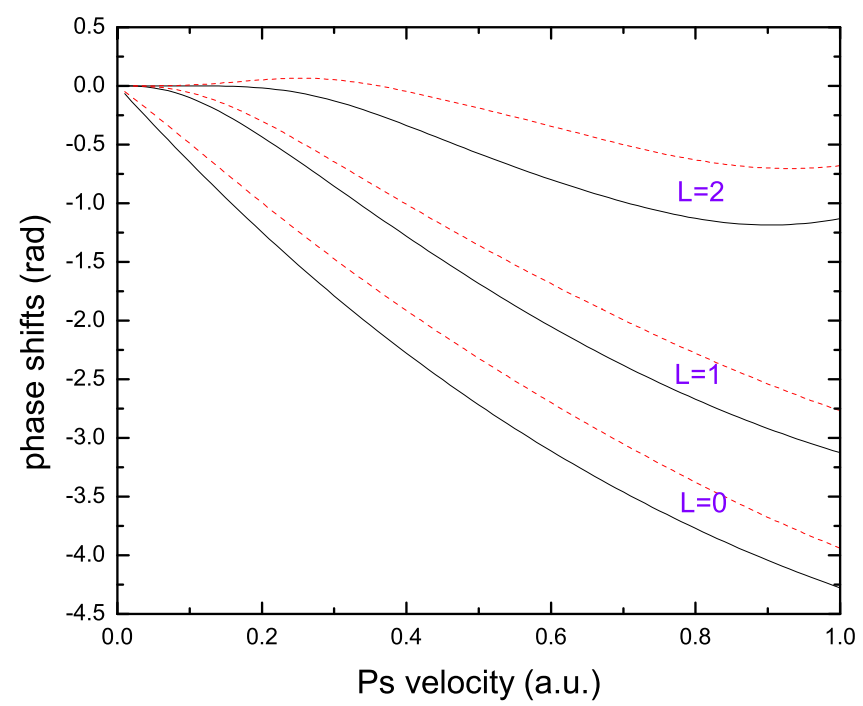

FIG. 3. Ps-Kr scattering phase shifts for $L=0,1$ and 2. For each partial wave, the lower curve (solid black) is for the static pseudopotential and the upper curve (dashed red) includes the effect of the van der Waals interaction with $C_{6}=152$ a.u. and $R_{c}=3.0$ a.u.

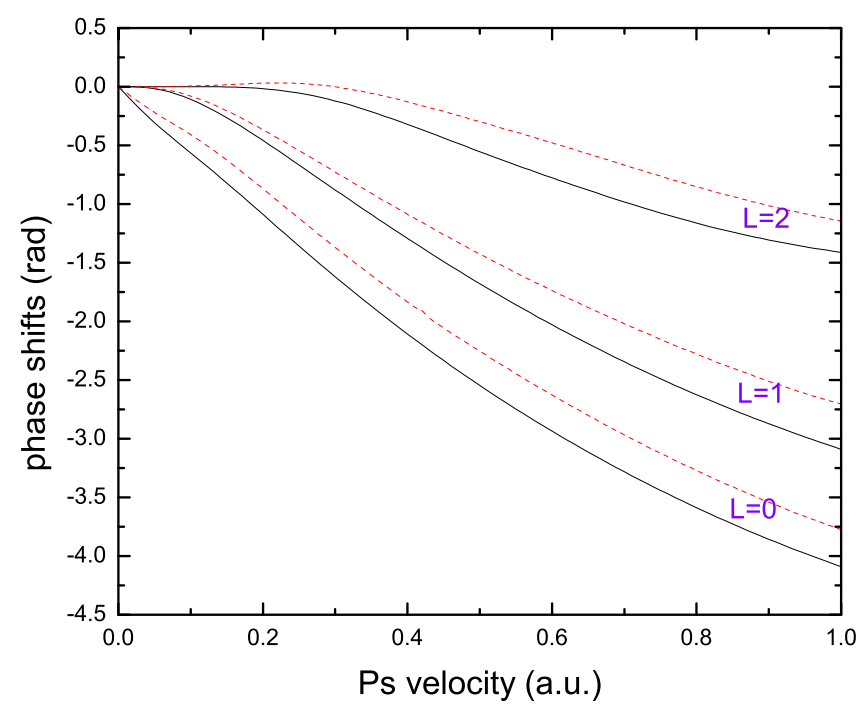

FIG. 8. Ps-Ar scattering phase shifts. For each $L$, the lower curve (solid black) was calculated using the static-field pseudopotential, while the upper (dashed red) also includes the effect of the van der Waals interaction with $C_{6}=104.52$ a.u. and $R_{c}=3.0$ a.u. 